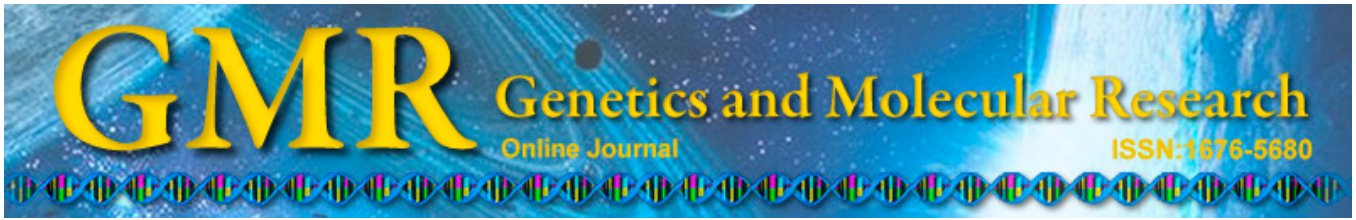

\title{
Expression and clinical value of the soluble major histocompatibility complex class I-related chain A molecule in the serum of patients with renal tumors
}

\author{
Y.-K. Zhao ${ }^{1 *}$, C.-M. Jia ${ }^{2 *}$, G.-J. Yuan ${ }^{1}$, W. Liu ${ }^{1}$, Y. Qiu ${ }^{1}$ and Q.-G. Zhu ${ }^{1}$ \\ ${ }^{1}$ Department of Urinary Surgery, \\ The Second Affiliated Hospital of Harbin Medical University, Harbin, China \\ ${ }^{2}$ Department of Hematology, Harbin Medical University Tumor Hospital, \\ Harbin, China \\ *These authors contributed equally to this study. \\ Corresponding author: Q.-G. Zhu \\ E-mail: zhuqingguo123@163.com
}

Genet. Mol. Res. 14 (2): 7233-7240 (2015)

Received September 30, 2014

Accepted January 21, 2015

Published June 29, 2015

DOI http://dx.doi.org/10.4238/2015.June.29.16

\begin{abstract}
We investigated the expression and clinical value of the soluble major histocompatibility complex class I-related chain A (sMICA) molecule in the serum of patients with renal tumors. Sixty patients diagnosed with renal tumors were enrolled in the experimental group, whereas 20 healthy volunteers served as the control group. The sMICA levels were measured via enzyme-linked immunosorbent assay, and the results were analyzed in combination with data from pathology examination. The experimental group had a statistically significant higher sMICA level $(\mathrm{P}<0.05)$ than the control group. The sMICA level was higher in patients with malignant tumors than in those with benign tumors. We also observed a positive relationship among different tumor-node-metastasis (TNM) pathological stages with more advanced
\end{abstract}


diseases exhibiting higher sMICA levels. As a tumor-associated antigen, MICA has a close relationship with renal tumorigenesis and immune escape. Our results indicated that sMICA levels were related to tumor pathology, TNM stage, and metastasis. Therefore, sMICA might be a potential marker for tumor characteristics, prognosis, and recurrence prediction.

Key words: Renal tumor; MICA; NK cell

\section{INTRODUCTION}

Urothelial carcinoma is a common disease of the urinary system, with bladder cancer having an especially high occurrence. The incidence of urothelial carcinoma has increased in recent years, possibly owing to the increased awareness by those performing physical examinations and the improvements of ultrasonography techniques. However, the disease's pathogenesis remains unclear. Recent studies have suggested that the human major histocompatibility complex class I-related chain A (MICA) molecule is highly expressed in epithelial cell-derived tumors (Pende et al., 2002). The binding of MICA to the natural-killer group 2, member D (NKG2D) receptor on the surface of NK cells can activate their killing effect, which plays an important role in tumor initiation and development. Carcinogenesis occurs when normal cells are stimulated by inflammation, viruses, or other factors. Such an event results in the genetic expression of MICA in cells, leading to an increased presence of MICA molecules on the cell surface. MICA molecules can be identified by NK cells, can bind to NKG2D receptors on the surface of NK cells, and activate NK cells to exert cytotoxic effects on tumor cells, resulting in tumor cell lysis. Therefore, MICA has an important function in early immune surveillance and elimination of tumor cells. Once a tumor has developed, the binding of NK cells and MICA molecules on the surface of cancer cells could inhibit their rapid growth.

Although in-depth studies have investigated the role of MICA in other epithelial tumors, its expression in urothelial carcinoma remains unclear. In the present study, we assessed MICA expression in 100 urothelial cancer patients admitted to our hospital between June and October 2012 to understand the relationship between MICA and the occurrence of urothelial carcinoma.

\section{MATERIAL AND METHODS}

\section{Study subjects}

One hundred patients with urothelial carcinoma were admitted to our hospital between June and August 2011, including 35 with renal pelvic tumors, 40 with bladder cancer, and 25 with ureteral tumors (Table 1). A total of 30 healthy volunteers, including 14 men and 16 women between 45 and 70 years of age (average, 52.3 years), were also recruited.

\section{Specimen collection}

Participants provided $2 \mathrm{~mL}$ peripheral venous blood in the morning after fasting. Each blood sample was collected in a test tube without anticoagulant, and centrifuged at 3000-3300 revolutions per minute for 10 min within 30 min after collection. The serum was then transferred to a $1.5-\mathrm{mL}$ Eppendorf tube and stored at $-20^{\circ} \mathrm{C}$ for analysis. 
Table 1. Clinical data of 100 urothelial cancer cases.

\begin{tabular}{lcccc}
\hline Body part & Gender (male/female, N) & Age (years) & Lymph node metastasis (yes/no, N) & Organ metastasis (yes/no, N) \\
\hline Pelvis & $20 / 15$ & $42-76$ & $8 / 27$ & $4 / 31$ \\
Ureter & $13 / 12$ & $45-72$ & $4 / 21$ & $3 / 22$ \\
Bladder & $21 / 19$ & $54-68$ & $3 / 37$ & $4 / 36$ \\
\hline
\end{tabular}

\section{Enzyme-linked immunosorbent assay (ELISA)}

ELISA kit was purchased from Wuhan Boster Biological Technology Ltd. According to the manufacturer protocol, $100 \mu \mathrm{L}$ standard or sample was added to the bottom of a well on a 96-well ELISA plate. One well was kept empty to serve as the control sample, and the two-well parallel pipetting method was used. The reaction was incubated at $37^{\circ} \mathrm{C}$ for $90 \mathrm{~min}$, after which $100 \mu \mathrm{L}$ biotin-antibody was added. The reaction was incubated at $37^{\circ} \mathrm{C}$ for $60 \mathrm{~min}$, and then washed three times with $0.01 \mathrm{MPBS}$ solution. After addition of $100 \mu \mathrm{L}$ ABC solution, the plate was incubated at $37^{\circ} \mathrm{C}$ for $30 \mathrm{~min}$ and then washed five times with $0.01 \mathrm{MPBS}$ solution. Subsequently, $100 \mu \mathrm{L}$ TMB solution was added, and the plate was incubated at $37^{\circ} \mathrm{C}$ for 15 to 20 min before reaction termination. The ELISA plate was immediately measured at $450 \mathrm{~nm}$ using an automatic microplate reader. The standard curve was plotted, and the actual concentration was calculated.

\section{Immunohistochemistry}

Urothelial carcinoma and normal urothelial tissue samples were collected during surgical resection. They were immediately soaked in formalin solution for 48 to $72 \mathrm{~h}$ to stabilize. After a series of processing steps, including dehydration and embedding, the samples were treated with goat anti-human primary antibody and rabbit anti-human secondary antibody. The expression of MICA molecule on the surface of urothelial cancer cells and normal urothelial cells was determined under an optical microscope.

\section{Real-time quantitative polymerase chain reaction (PCR)}

Nine urothelial carcinoma and three normal urothelial tissue specimens were collected via surgical resection, and immediately placed in liquid nitrogen. Samples of approximately $0.5 \mathrm{~cm}$ in diameter were homogenized. Trizol reagent was used to extract RNA from these samples. Real-time quantitative PCR was performed to determine mRNA expression levels of MICA in tumor tissues and tissues adjacent to the tumor. The primer sequences and sizes are shown in Table 2.

Table 2. Sequences and sizes of MICA mRNA primers.

\begin{tabular}{llr}
\hline Name & Sequence & Size (bp) \\
\hline Primer MICA F & ACACAGCGGGAATCACAGCACTC & 80 \\
Primer MICA R & CATGGAATGTCTGCCAATGACTCTG & 80 \\
Human ACTIN F & ACTTAGTTGCGTTACACCCTT & 156 \\
Human ACTIN R & ACTTAGTTGCGTTACACCCTT & 156 \\
\hline
\end{tabular}

MICA, major histocompatibility complex class I-related chain A. 


\section{Statistical analysis}

The SPSS statistical software was used for statistical analysis of all experimental data. The levels of soluble MICA (sMICA) for renal cancer patients and healthy controls were compared using the $t$-test, as were the serum sMICA levels of patients at different tumor-nodemetastasis stages and various metastatic degrees.

\section{RESULTS}

\section{Comparison of serum sMICA levels in the urothelial carcinoma and healthy control groups}

The serum sMICA levels were significantly higher in urothelial carcinoma patients than in healthy controls $(\mathrm{P}<0.05$, Table 3$)$. However, no significant differences in serum sMICA levels were observed among patients with renal pelvis cancer, ureter cancer, or bladder cancer $(\mathrm{P}>0.05)$. The bladder cancer group was further stratified into initial occurrence and recurrence. Recurrence bladder cancer patients had higher sMICA levels than those with initial occurrence $(\mathrm{P}<0.05)$. Furthermore, sMICA levels in patients with multiple occurrence bladder cancer were higher than those in single occurrence patients $(\mathrm{P}<0.05)$. There was no significant difference in SMICA levels between patients with and without lymph node metastasis $(\mathrm{P}>0.05)$, whereas sMICA levels were higher in patients with organ metastasis than in those without $(\mathrm{P}<0.05$, Table 4$)$.

\begin{tabular}{lccc}
\multicolumn{4}{c}{ Table 3. sMICA levels in the renal cancer and healthy control groups. } \\
\hline Group & Cases & sMICA $(\mathrm{pg} / \mathrm{mL})$ & P \\
\hline Pelvis & 35 & $318.5 \pm 32.5$ & \\
Ureter & 25 & $309.3 \pm 30.4$ & $<0.05$ \\
Bladder & 40 & $321.5 \pm 23.5$ & $168.4 \pm 43.2$ \\
Healthy control & 30 & &
\end{tabular}

sMICA, soluble major histocompatibility complex class I-related chain A.

\section{Expression of the MICA molecule on the surface of urothelial cancer cell and normal urothelial cells}

Higher expression of MICA molecules was observed on the surface of urothelial cancer cells surface than on the surface of normal urothelial cells (Figure 1).

\section{MICA mRNA expression in tumor cells and those adjacent to a tumor}

Real-time quantitative PCR results revealed that MICA mRNA expression in urothelial carcinoma cells was significantly higher than that in normal urothelial cells $(\mathrm{P}<0.05$, Table 5). However, no significant differences in MICA mRNA levels were observed among urothelial cells of the pelvis, ureter, and bladder cancer groups $(\mathrm{P}>0.05)$. 
Table 4. sMICA serum levels and metastasis of urothelial carcinoma.

\begin{tabular}{llccc}
\hline Group & & sMICA $(\mathrm{pg} / \mathrm{mL})$ & Case & P \\
\hline Pelvis group & & & 35 & $<0.05$ \\
Lymph node metastasis & No & $314.5 \pm 27.5$ & 27 & \\
Organ metastasis & Yes & $328.5 \pm 32.5$ & 8 & $<0.05$ \\
& No & $310.5 \pm 27.5$ & 31 & \\
Ureter group & Yes & $327.3 \pm 28.2$ & 4 & $>0.05$ \\
Lymph node metastasis & No & $305.3 \pm 28.4$ & 25 & \\
Organ metastasis & Yes & $312.4 \pm 32.1$ & 4 & $<0.05$ \\
& No & $308.2 \pm 29.4$ & & \\
Bladder group & Yes & $326.3 \pm 28.4$ & & $<0.05$ \\
& First occurrence & $305.5 \pm 21.5$ & 25 & $>0.05$ \\
Lymph node metastasis & Re-occurrence & $326.3 \pm 22.6$ & 15 & \\
& Single occurrence & $316.5 \pm 21.5$ & 17 & $>0.05$ \\
Organ metastasis & Multiple occurrences & $328.5 \pm 22.3$ & 3 & \\
& Yes & $324.5 \pm 23.5$ & 37 & \\
& No & $316.2 \pm 22.4$ & 3 & \\
\hline
\end{tabular}

sMICA, soluble major histocompatibility complex class I-related chain A.

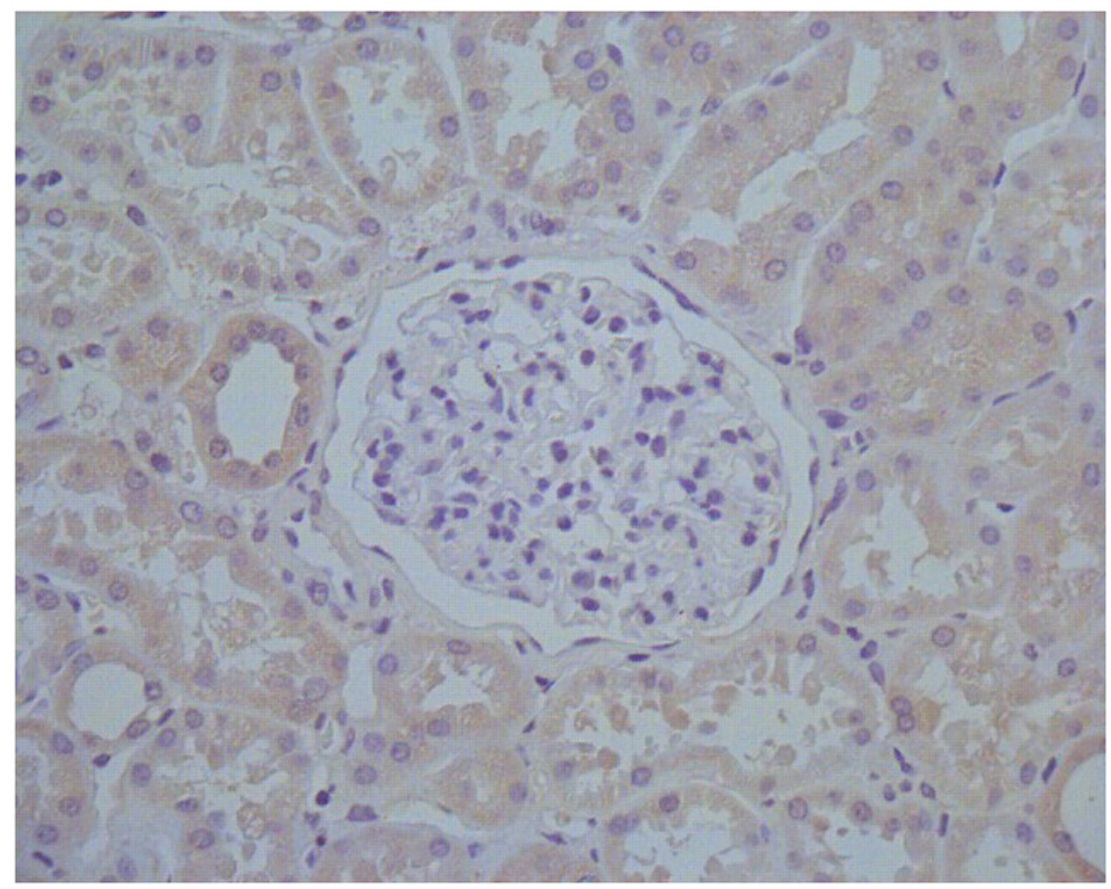

Figure 1. Immunohistochemical staining of urothelial carcinoma, 400X magnification. 
Table 5. Relative expression of MICA mRNA in urothelial carcinoma cells and normal urothelial cells.

\begin{tabular}{lcc}
\hline Sample labels & Average $($ Mean \pm SD) & $P$ \\
\hline Pelvis group & $2.0313 \pm 0.7514$ & \\
Ureter group & $2.0294 \pm 0.7423$ & \\
Bladder group & $2.0279 \pm 0.7425$ & $<0.05$ \\
Control group & $0.7668 \pm 0.2121$ &
\end{tabular}

MICA, major histocompatibility complex class I-related chain A.

\section{DISCUSSION}

The MICA gene was first discovered in 1994 (Bahram et al., 1994). It is located 46.4 $\mathrm{kb}$ away from the human leukocyte antigen B (HLA-B) locus, and its encoded product is a $620-\mathrm{kDa}$ cell surface glycoprotein with similar structure to that of HLA. MICA antigens are normally expressed on the surface of endothelial cells, epithelial cells, and cells of the gastrointestinal tract, but they are not expressed in peripheral blood lymphocytes and other normal tissues. MICA molecules can bind as a ligand to NKG2D receptors on the surface of NK cells to activate their cytotoxic function, leading to cell death via lysis. NKG2D receptors are present on the surface of NK cells, CD $8+\alpha \beta T$ cells, $\gamma \delta \mathrm{T}$ cells, and macrophages. The receptor is a member of the C-type lectin superfamily (Bauer et al., 1999). Alternatively, the binding of inhibitory receptors on the surface of NK cells to major histocompatibility complex class I molecules on the surface of normal cells might inhibit the activity of NK cells, resulting in a negative regulation that helps avoid normal cell attack (Campbell and Colonna, 2001). When the amount of inhibitory ligands on the surface of normal cells decreases or that of MICA increases, these normal cells may become a target for NK cells. Since the expression of MICA molecules is associated with stress-induced factors, their increased expression can be induced by viral or bacterial infection. MICA expression is observed in many epithelial cell-derived tumors such as colon cancer, breast cancer, and lung cancer (Vallian et al., 2012; Zhang et al., 2012; Chen et al., 2013), and on the surface of some melanoma cells. Renal cancer cells also express MICA (Qiu et al., 2013). Thus, MICA is known as a tumor-associated antigen (Groh et al., 1999). MICA molecules can be divided into two types according to their location: membrane MICA (mMICA), which exists on the surface of tumor cells, and sMICA, which is present in the serum. The latter is formed after MICA is released from the surface of tumor cells into the serum owing to the effects of matrix metalloproteinases.

\section{Differences in SMICA expression in the serum of urothelial carcinoma patients and healthy controls}

Serum sMICA levels of urothelial carcinoma patients were significantly higher than those of normal individuals $(\mathrm{P}<0.05)$. sMICA levels in patients with renal pelvis cancer, ureter cancer, and bladder cancer did not significantly differ $(\mathrm{P}>0.05)$. The bladder cancer group was further divided into patients with initial disease occurrence or recurrence, and no significant differences in sMICA levels were observed between the two sub-groups $(\mathrm{P}>0.05)$. Such a result might be due to the similarity in tumor types, because pelvis, ureter, and bladder epithelial cells are all urothelial cells. The sMICA levels in patients with multiple occurrences of bladder cancer did not significantly differ from those in patients with a single disease occurrence $(\mathrm{P}>0.05)$, suggesting that the sMICA level might not be associated with the number of 
tumors. Recurrences of bladder cancer showed higher sMICA levels than the first occurrences $(\mathrm{P}<0.05)$. In general, the prognoses for pelvis and ureter cancer are relatively poor, whereas that for bladder cancer is better. Such a difference might be associated with the metastatic potential via the lymphatic system in pelvis and ureter cancer. We compared serum sMICA levels in bladder, pelvis, and ureter cancer patients with and without lymph node or organ metastasis, and found an association between sMICA levels and organ metastasis $(\mathrm{P}<0.05)$ but not lymph node metastasis $(\mathrm{P}>0.05)$. The prognosis of recurrent bladder cancer is generally poorer than that of the first disease occurrence, indicating a possible positive link between serum sMICA levels and prognosis. Therefore, sMICA could be used as a predictive marker for patient prognosis.

\section{Differences in the expression of MICA molecules on the surface of urothelial cancer cells and normal urothelial cells}

MICA expression in normal urothelial cells is unclear. Our immunohistochemistry results demonstrated high MICA expression on the surface of tumor cells and low expression in cells adjacent to a tumor. Such a result indicated that MICA expression increased after urothelial cell transformation, and that MICA molecules might be associated with tumor development and progression.

\section{Differences in MICA mRNA expression in urothelial cancer cells and normal urothelial cells}

Real-time quantitative PCR was performed to detect MICA gene transcription in urothelial cancer cells and normal urothelial cells. MICA mRNA expression in urothelial carcinoma cells was significantly higher than that in normal urothelial cells $(\mathrm{P}<0.05)$, indicating that MICA gene transcription increased upon activation by carcinogenic factors. However, the carcinogenic factors and mechanisms that induce such an increase remain unclear. The increase of MICA gene expression might lead to a rise in antigen expression on the surface of cancer cells, allowing them to be identified and eliminated by immune cells.

A precondition for NK cells to exert lethal effects on tumor cells is the binding of mMICA molecules on the surface of cancer cells. Metalloenzymes transform mMICA conformation during cancer cell development, resulting in the release of mMICA from the surface of tumor cells into the serum as sMICA molecules. As the formation of sMICA molecules progresses, the number of mMICA molecules on the surface of tumor cells decreases, reducing the possibility of identification and cytotoxic effects by NK cells. On the other hand, the binding of sMICA molecules to NK cells not only inhibits that of mMICA but also down-regulates NKG2D receptor expression on the surface of NK cells (Doubrovina et al., 2003). Therefore, the formation of sMICA molecules is considered a sign of tumor progression and a mechanism for tumor metastasis via immune evasion.

The association between MICA, NK cells, and tumor has become a popular topic in cancer research owing to the unique relationship between MICA and NK cells. Previous studies include the use of MICA antibodies to block MICA expression on the surface of tumor cells, addition of sMICA to reduce the lethal effects of NK cells on tumor cells, or utilization of metalloenzymes to enhance the lethal effect of NK cells on tumor cells (Salih et al., 2002). These reports indicate that increased expression of mMICA on the surface of tumor cells and 
decreased formation of sMICA could strengthen the lethal effect on tumor cells, and significantly inhibit tumor growth. On the basis of such observations, Germain et al. (2005) genetically combined the Fab' fragment of anti-carcinoembryonic antigen (CEA) with reconstructed MICA. The binding site of anti-CEA was the tumor cell, and that of MICA was NKG2D, which effectively activated the lethal activity of NK cells.

The relationship between MICA and urothelial carcinoma is poorly understood with many uncertainties. Nonetheless, MICA, an urothelial carcinoma-associated antigen closely associated with urothelial carcinoma occurrence and development, undoubtedly provides a new explanation of the mechanisms of tumorigenesis and a potential treatment pathway.

\section{Conflicts of interest}

The authors declare no conflict of interest.

\section{ACKNOWLEDGMENTS}

Research supported by the Wu Jieping Medical Foundation.

\section{REFERENCES}

Bahram S, Bresnahan M, Geraghty DE and Spies T (1994). A second lineage of mammalian major histocompatibility complex class I genes. Proc. Natl. Acad. Sci. U.S.A. 91: 6259-6263.

Bauer S, Groh V, Wu J, Steinle A, et al. (1999). Activation of NK cells and T cells by NKG2D, a receptor for stressinducible MICA. Science 285: 727-729.

Campbell KS and Colonna M (2001). Human natural killer cell receptors and signal transduction. Int. Rev. Immunol. 20: 333-370.

Chen Y, Lin G, Guo ZQ, Zhou ZF, et al. (2013). Effects of MICA expression on the prognosis of advanced non-small cell lung cancer and the efficacy of CIK therapy. PLoS One 8: e69044.

Doubrovina ES, Doubrovin MM, Vider E, Sisson RB, et al. (2003). Evasion from NK cell immunity by MHC class I chainrelated molecules expressing colon adenocarcinoma. J Immunol. 171: 6891-6899.

Germain C, Larbouret C, Cesson V, Donda A, et al. (2005). MHC class I-related chain A conjugated to antitumor antibodies can sensitize tumor cells to specific lysis by natural killer cells. Clin. Cancer Res. 11: 7516-7522.

Groh V, Rhinehart R, Secrist H, Bauer S, et al. (1999). Broad tumor-associated expression and recognition by tumorderived gamma delta T cells of MICA and MICB. Proc. Natl. Acad. Sci. U.S.A. 96: 6879-6884.

Pende D, Rivera P, Marcenaro S, Chang CC, et al. (2002). Major histocompatibility complex class I-related chain A and UL16-binding protein expression on tumor cell lines of different histotypes: analysis of tumor susceptibility to NKG2D-dependent natural killer cell cytotoxicity. Cancer Res. 62: 6178-6186.

Qiu Y, Zhao YK, Yuan GJ and Zhu QG (2013). Clinical significance of soluble major histocompatibility complex class I chain-related a in renal cell carcinoma patients. Asian Pac. J. Cancer Prev. 14: 5651-5655.

Salih HR, Rammensee HG and Steinle A (2002). Cutting edge: down-regulation of MICA on human tumors by proteolytic shedding. J. Immunol. 169: 4098-4102.

Vallian S, Rad MJ, Tavallaei M and Tavassoli M (2012). Correlation of major histocompatibility complex class I related A (MICA) polymorphism with the risk of developing breast cancer. Med. Oncol. 29: 5-9.

Zhang Z, Su T, He L, Wang H, et al. (2012). Identification and functional analysis of ligands for natural killer cell activating receptors in colon carcinoma. Tohoku J. Exp. Med. 226: 59-68. 